\title{
The Potential for Excess Correlation (Entanglement) between Flow States in Pairs of Gamers Sharing Specific Circumcerebral Rotating Magnetic Fields
}

\author{
Brendan S. Lehman, Michael A. Persinger \\ Behavioural Neuroscience Program and Department of Biology, Laurentian University, Sudbury, Canada \\ Email: bx_lehman@laurentian.ca, mpersinger@laurentian.ca
}

Received 3 April 2016; accepted 15 July 2016; published 18 July 2016

Copyright (C) 2016 by authors and Scientific Research Publishing Inc. This work is licensed under the Creative Commons Attribution International License (CC BY). http://creativecommons.org/licenses/by/4.0/ (c) ()) Open Access

\section{Abstract}

Excess correlation between the activity or properties of two particles separated by non-local distances has been demonstrated for photons, shifts in relative proton and hydroxyl ratios in spring water, and the distribution of values from random number generators if both loci shared a specific type of rotational magnetic field. Previous experiments had shown that specific responses from pairs of people who shared circumcerebral magnetic fields with changing angular velocities revealed significant excess correlation. The most significant differences occurred during the component of the field exposure that has previously been associated with "excess correlation". In the present experiments, we found evidence of excess correlation of performance (serial in-game scores) occurred between pairs (separated by $10 \mathrm{~m}$ ) of experienced gamers during the relative measures for the central portion of the protocol but was diminished when the "excess correlation" electromagnetic fields were activated, suggesting a similar competing mechanism. The results are consistent with the interpretation that shared video systems and activities may enhance excess correlation of responses. This can be simulated in novice players by experimentally inducing excess correlation through appropriate application of changing, circumcerebral angular velocity magnetic fields that were similar in magnitude to those associated with computer systems and time frames that define human consciousness.

\section{Keywords}

Excess Correlation, Entanglement, Digital Games, Learning, Magnetic Fields, Consciousness 


\section{Introduction}

According to Aczel [1], entanglement is one of the greatest mysteries in physics. The empirical demonstration of entanglement requires an excess correlation between the responses of two units in non-local space. Non-local space means that the mutual responses of the two units occur over any distance (and potentially any time) and hence are not limited to the mechanisms required for locality. The latter can only occur if there is a medium through which a force or energy can be mediated and consequently diminishes in some systematic manner as a function of the distance between the two units. Excess correlation means that over the measured time increments the correlation between the properties such as magnitudes or variations of frequency (including phase modulation) occurs when only random relationships should exist according to the assumptions of locality.

The general consensus is that excess correlation, or by inference entanglement, can occur anywhere in space and primarily involves photons. For example, changing the parity of one photon in a pair that has been entangled, usually because of initial proximity from their shared source, results in the opposite change in the other photon within the pair. There is also evidence of excess correlation at the macroscopic level. Dotta and Persinger [2] found that when two chemiluminescent reactions shared the same circular-rotating magnetic fields that exhibited specific uncoupling between the group and phase velocities, the injection of the reactant into both solutions simultaneously resulted in a doubling of the photon emissions. In other words, the excess correlation behaved as if the two non-local spaces and been transiently superimposed into the same locus such that the reaction was twice as intense.

Murugan, Karbowski, Dotta, and Persinger [3] later showed that two beakers of water separated by non-local distances but sharing the same "excess correlation" patterned magnetic fields also exhibited entanglement. When a small proton source was introduced into one beaker of spring water, producing an increase in acidity, there was a drift in the other beaker of spring water, within which nothing had been injected, towards the alkaline condition. The shift in $\mathrm{pH}$ in the non-local site was proportional to the numbers of hydronium ions that would be affected by the energy available from the intensity of the applied magnetic field within that volume of water. In all of these experiments there were two phases of the entanglement paradigm. The first was considered a "primer" while the second, the "effector," was considered the actual demonstration of entanglement. If the primer was not delivered there was no evidence of excess correlation.

That human brains can display entanglement has recently been demonstrated by Scott, et al. [4]. They showed that pairs of people separated by $6000 \mathrm{~km}$ and exposed to the excess correlation paradigm showed clear evidence of coherence of quantitative brain activity but only during the effective component of the entanglement paradigm. In addition during the presentation of a specific tone to both participants, separated by $6000 \mathrm{~km}$ but sharing the same circumcerebral magnetic fields, the shift in spectral power density within both cerebrums of a pair was in the opposite direction. Although both displayed the $7 \mathrm{~Hz}$ power shift associated with pulsing of the sound stimulus, one of each pair displayed increased spectral density power while the other showed diminished spectral density power at the same time.

Entanglement requires conditions in which two units share some property in space-time such that later when the two objects are separated there is a residual of this shared property in each. They create a functional system [5]. Activation of this system allows both units to interact regardless of distance. By the nature of computer (electronic) games, which are relatively repetitive due to their programming, pairs of gamers might be exposed intermittently to entanglement-inducing condition. Consequently, even at great distances the behaviours of one gamer could exhibit excess correlation over time with the behaviors of another gamer. The two might not be aware of this excess correlation. If the events occurred frequently enough one might imagine the extent of the excess correlation to generalize to other aspects of their behavior.

Spontaneous occurrences are difficult to control, particularly when the causal factors are not clear. Experimental manipulations whereby the essential elements of the condition, in this case entanglement, are created in the laboratory can enhance the magnitude of the effect in order for direct discernment to be possible. This procedure might be considered equivalent to isolating the essential ingredient of white willow bark, acetylsalicylic acid, in order to produce reliable analgesia and antipyretic effects. In the present study, the potentially enhancing effects of the excess correlation paradigm that has been shown for $\mathrm{pH}$ and photon reactions was applied to the brains of pairs of experienced or novice gamers.

We predicted that the entanglement paradigm should simulate the condition of paired experienced gamers and thus produce coherence in a paired novice similar to that of experienced gamers. On the other hand, if the gamer 
connection is related to unique entanglement the novel excess correlation field exposure should disrupt this connection much like a competitive antagonist for drug action. For the experimental entanglement between two experimenters to be feasible and practical, we assumed the effect size must be large enough to be evident with a small sample size.

\section{Materials \& Methods}

\subsection{Participants}

Following approval of the university's research ethics board, a total of 7 identified male participants (ages between 24 and 32 years) participated in the study. Both A and B field application condition groups and positive control groups involved pairs or either one novice and one experienced player, or two experienced players. Experience was established by the participant reported having played the game prior to the experiment for at least 3 hours. Novice designation was given to participants with no prior exposure to the game used. Negative control groups involved one novice player alone, external to either of the field application chambers. This was implemented to control for space-memory effects.

\subsection{Interactive Stimuli and Performance Metrics}

Use of a complex stimulus involved play of the video game Super Hexagon [6]. The game was played on either a Lenovo Y580 or Asus UX31 laptop. The game involves the movement of a radius-fixed cursor in a circular pattern to avoid obstacles (Figure 1) using only the left and right arrow keys. Obstacles rotate in either clockwise or counterclockwise direction around the centre point of the screen, moving from the outer edges of the screen toward the centre. The shape and pattern of oncoming obstacles increases in complexity as the stage increases, up to 60 seconds, where the speed and complexity increase further. The "death" condition consisted of contacting an obstacle, where the play time of that "run" would be displayed. Pressing the space bar starts a new run, resetting to the beginning. The game was run on the standard "hard" setting. Performance was measured using the game timer, whose large unit counted in seconds and sub-second unit counts up to 60. Each 10 seconds reached a milestone, where obstacle shape and puzzle complexity increased.

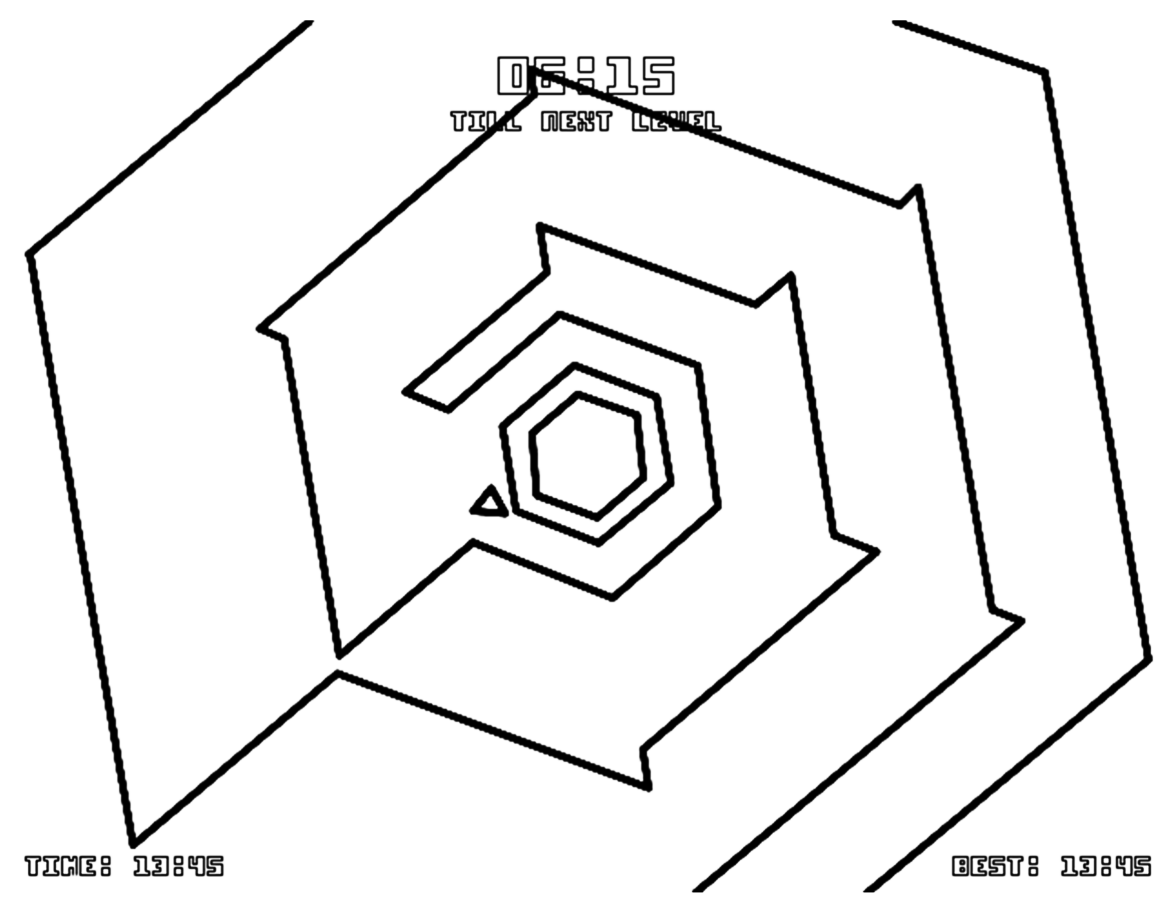

Figure 1. Simplified user interface example of Super Hexagon. The cursor rotates at a fixed radius from the centre with player input, avoiding oncoming obstacles. The current "run" time is displayed in the top centre with a progress bar to the next stage. The best time is displayed in the top left. 


\subsection{Design of Experimental Apparatus}

The paradigm, as shown in Figure 2, utilized the same equipment and procedures employed in previous human brain studies [7]. Details of exposure procedures have been published elsewhere [2]. In summary, two identical circular (55 - $60 \mathrm{~cm}$ circumference) arrangements of 8 equally spaced pairs of solenoids ( $45^{\circ}$ separation) were placed in two separate rooms separated by $10 \mathrm{~m}$. The pairs of solenoids were reed switches connected to custom constructed equipment (Canadian Patent 2,214,296). The two devices were connected to the same Intel 286 computer housed in a room separate from the rooms containing the circumcerebralcoils. This computer controlled the timing and amplitude delivery of the current delivered to the solenoids to generate the magnetic fields. This DOS-based system produces reliable inter-experiment output point durations to insure that the current durations (and hence magnetic fields) are not affected by competing software operations, and are always based upon actual 1 ms intervals.

Custom software (Complex Software ${ }^{\odot}$ ) was programmable to generate the applied pattern at each solenoid for different durations. A temporal configuration of “20-2" indicated that the 1st (over the left prefrontal regions) solenoid received the field pattern for $20 \mathrm{~ms}$, the next solenoid received the pattern for $22 \mathrm{~ms}$, etc., until the last (8th) solenoid received the pattern for $34 \mathrm{~ms}$ before the cycle began again in the 1st solenoid (sum = 216 ms or 4.6 cycles/s). The duration of $20 \mathrm{~ms}$ was based upon the theoretical assumption of the "cohesive binding" factor for consciousness as described by Edelman [8] and Llinas and Ribardy [9]. A study by Cook, Koren, and Persinger [10] showed that the most significant distortion of subjective time estimation was produced by counterclockwise (as viewed from the top) circumcerebral magnetic fields, and has remained the standard in this regard.

The two patterns that were generated to each pair of solenoids were selected from the two shapes that have been most robust in producing changes in both the experiences and quantitative EEG patterns in human subjects [7] [11] [12]. Hallmarks of the two patterns were a decelerating frequency modulation ("Thomas pulse,” named after a previous student) and an accelerating frequency modulation ("burst firing”). Their shapes have been published with previous studies [11] [13]. These patterns were generated by converting a series (columns) of numbers between 0 and 256 (below 127 is negative polarity, above 127 is positive polarity) within the Complex Software $^{\odot}$ to between -5 and $+5 \mathrm{~V}$ through custom-constructed digital-to-analog converters (DAC). The strength of the magnetic fields as measured by a Metex meter coupled to a magnetic field sensor (EFM 140-3-60-1499) along the inside of each pair of solenoids was 1 to $5 \mu \mathrm{T}$ with the average within the cross-sectional area of approximately 1 to $2 \mu \mathrm{T}$.

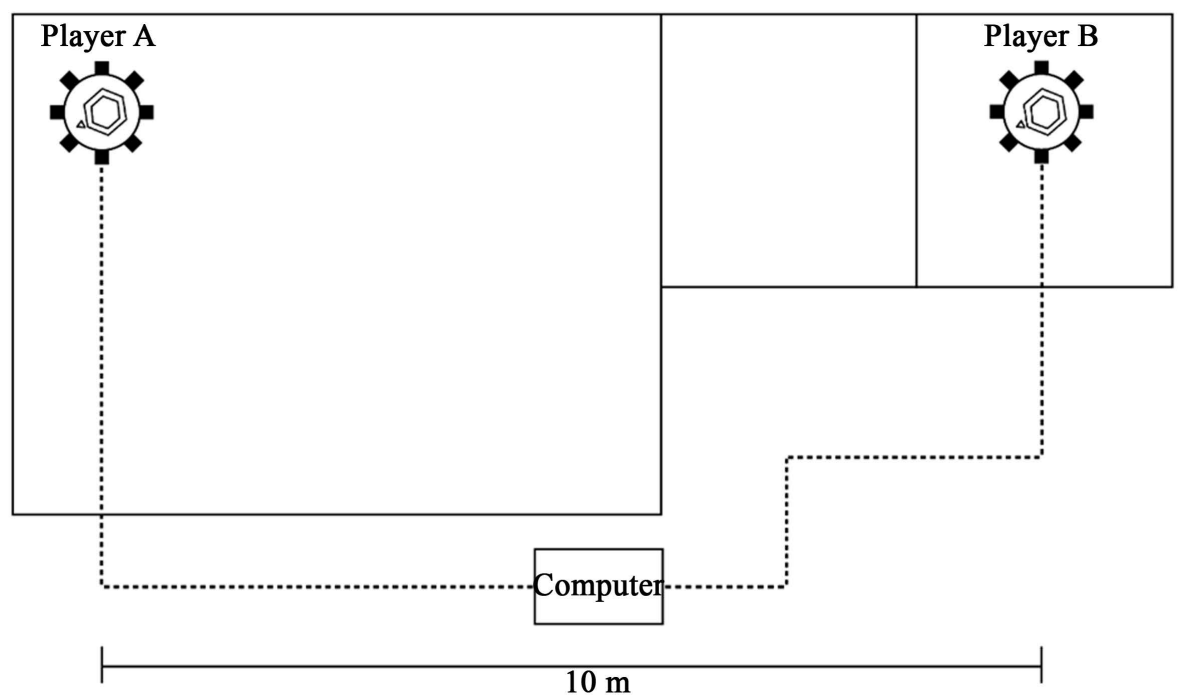

Figure 2. Diagram of procedure by which the same magnetic field condition was generated in two separate spaces separated by about $10 \mathrm{~m}$. The two circular arrays represent the 8 pairs of solenoids. The magnetic fields were generated as brief pulses whose durations either increased or decreased as the programmable fields were rotated around the solenoids in a counterclockwise direction. The pattern of the magnetic field that was generated reflected either a decreasing or increasing frequency or "phase" modulation. 


\subsection{Experimental Procedure: Field Application}

The all field application condition groups consisted of two players. All field application condition groups were exposed in two rooms. The first room was an acoustic chamber and Faraday cage [13]. Players were comfortably seated in a cushioned chair within the chamber with the laptop on their lap, facing in a north/south direction. The second room was a darkened room. The players were also seated in a comfortable chair, laptop on their laps, and facing a north/south direction. Data collection involved the assistance of two colleagues (one per player), who would record the run time called out by the player after each "death". To maintain temporal consistency, the start of each experimental session was synchronized between players and marked with a stopwatch. A new run was started by the call of the assistant every 2 minutes for the experienced players and 30 seconds for the novice players.

In the event a player remained playing at the end of the 2 minutes, they were instructed to force a death and to restart. At this point the assistant recorded " 2 minutes" as the run time. The session ended after 22 minutes (11 experienced runs). Application of field patterns was timed with these increments (changes performed by the chamber assistant); the first 2 runs being a pre-condition baseline (no field), the next 3 runs being the accelerating pattern, the next 4 runs being the decelerating pattern, and the final 2 runs being a post-condition baseline (no field). The A field application group involved two experienced players undergoing 4 trials, and the $\mathrm{B}$ group involved one experienced player and one novice utilizing the same procedure as described above for 3 different novices.

\subsection{Experimental Procedure: Control}

Control condition groups consisted of a positive and negative type. The positive control condition group was composed of both experienced/experienced pairs undergoing 3 trials, and experienced/novice pairs undergoing 1 trial with a novice from the field application condition group. All procedure for the positive control condition group were identical to the field application condition group with respect to environment, timing, and data collection, except without the application of the magnetic fields. The negative control condition group involved either one novice or one control, paired with one colleague for data collection, and included 1 trial each. This group was situated in a room external to both the field application condition rooms, but adjacent and within the $10 \mathrm{~m}$. This group used the Lenovo laptop to play the game as per the standard timing structure on a tabletop.

\subsection{Statistical Analysis}

Data collected by assistants were immediately entered and organized into Microsoft Excel for preliminary computation. All raw data was individually standardized using z-scores before any further statistical analyses. Data was detrended by subtracting the linear trend associated with learning (control comparison). Calculation of means, standard deviations, and standard error of the means were performed in Microsoft Excel 2013, and all oneway and correlation analyses were completed in SPSS Statistics 17 on a Windows-based computer.

\section{Results}

Direct comparison of field and no field exposure condition performance raw and z-scores for all players combined yielded no statistically significant differences. Comparison of the effects of exposure to magnetic fields on mean individual performance $\mathrm{z}$-scores over the length of the exposure paradigm for all experienced players combined returned a significantly lower field condition z-score in the pre-baseline condition and significantly higher field condition z-score in the BurstX condition (Figure 3(a); $F_{(3,32)}=4.58, p=0.01, \eta^{2}=0.32$ ). Comparison of all novice players combined returned no significant between field and no field groups. These procedures detrended z-scores for all experienced players combined highlighted elevated no field group values over the field group in the pre-baseline condition and elevated field group values over the no field group in the BurstX conditions. These were similar to the standard z-score results (Figure 4(a); $F_{(9,29)}=2.62, p=0.035, \eta^{2}=0.54$ ).

Such analysis for all novice players (combined) yielded higher field condition scores in the post-baseline condition, as with standard z-score results (Figure 3(b)). However ANOVA indicated no significant difference. Combining all players together yielded no significant differences between field exposed and no field groups. Direct comparison of combined field exposed novice and their paired experienced skill group individual performance z-scores yielded significantly higher scores for experienced players during the BurstX field exposure 


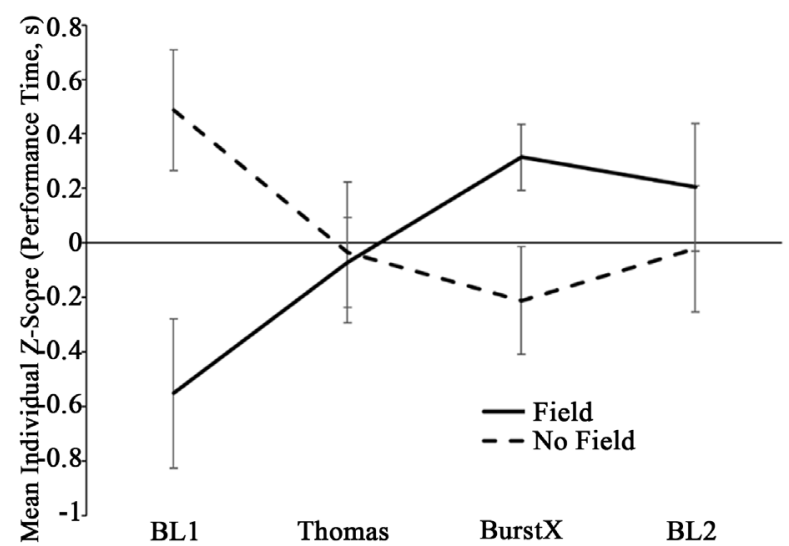

(a)

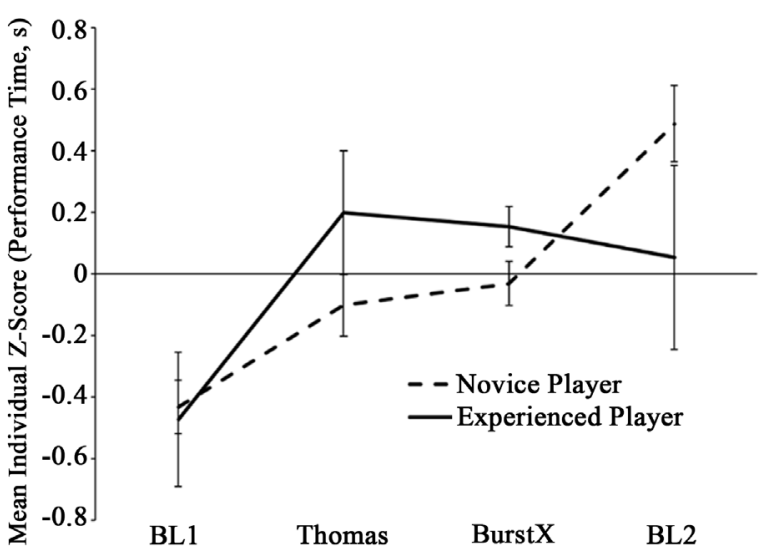

(b)

Figure 3. (a) Comparison of mean individual z-scores of performance time (in s) between experienced players exposed to fields and experienced players not exposed to fields. Error bars are SEM; (b) Comparison of mean individual z-scores of performance time (in s) between field exposed novice players and their paired experienced players. Error bars are SEM.

condition, and significantly higher scores for novice players during the post-baseline condition (Figure 4(b); $F_{(3,16)}$ $=3.83, p=0.036, \eta^{2}=0.37$ ).

\section{Discussion}

The concept of excess correlation predicts that if two complex systems have shared a space-time condition such that a subset of one is contained in the other as well as vice versa, a subsequent change in one of the two regardless of spatial separation should be associated with a complimentary change in the other. Juden-Kelly, Dotta, Vares, and Persinger [14] showed that when two RNG (random number generators) were each placed within a circular array of solenoids, identical to the system employed in the present study, a positive drift in one output was associated with a negative drift in the other.

However, this only occurred after both systems had been exposed to "primer" magnetic field which has a frequency modulated (descending frequency or phase modulation) known as the "Thomas pulse" that was contained within an accelerating angular velocity of field activation around the array of 8 solenoid pairs followed by the effector pattern which was frequency modulated (descending frequency of phase modulation) within a decelerating angular velocity system. These effects were evident, even within a small sample, within Figure 3 for experienced pairs.

As shown in Figure 4(a), the experienced pairs of players displayed enhanced coherence during the effector phase (when the excess correlation occurred in paired reactions of $\mathrm{pH}$ shifts, random number variations, and photon emissions) which was indicated by Figure 3(b). This was not observed in the novices. In that group, the effects of field occurred during the rebound interval of the post-baseline. One would expect that if excess correlations of the game performance (score) for two people playing the same game occurred naturally within seasoned players then applications of fields that enhance a similar process should be evident in others during the "excess correlation" period. Indeed that was measured. A particularly intriguing result occurred during the "rebound" periods of the entanglement sequence. As noted in Figure 4(c), there was a potential manifestation of the "parity" effect often observed in entanglement experiments. This occurs when the property exhibited by one of the pair is changed and the other member of the pair changes its property so that parity can be maintained. When the immediate pre- and post-baseline were compared with respect to standardized accuracy in the game, there was the equivalent of two standard deviations difference between two mirror conditions. The first was composed of the novice pairs exposed to the field and the experienced pair not exposed to the field. The second that was more than two z-scores less was composed of the novice pairs not exposed to the field and experienced pairs exposed to the field.

It is useful to describe this effect using an equilibrium metaphor. The game performance standard is set by the level of the most experienced player. This energy, by the principles of parity in a classic entanglement setting, moves to create a stasis between the players, and in doing so creates a synchrony (or trend toward it) of perfor- 

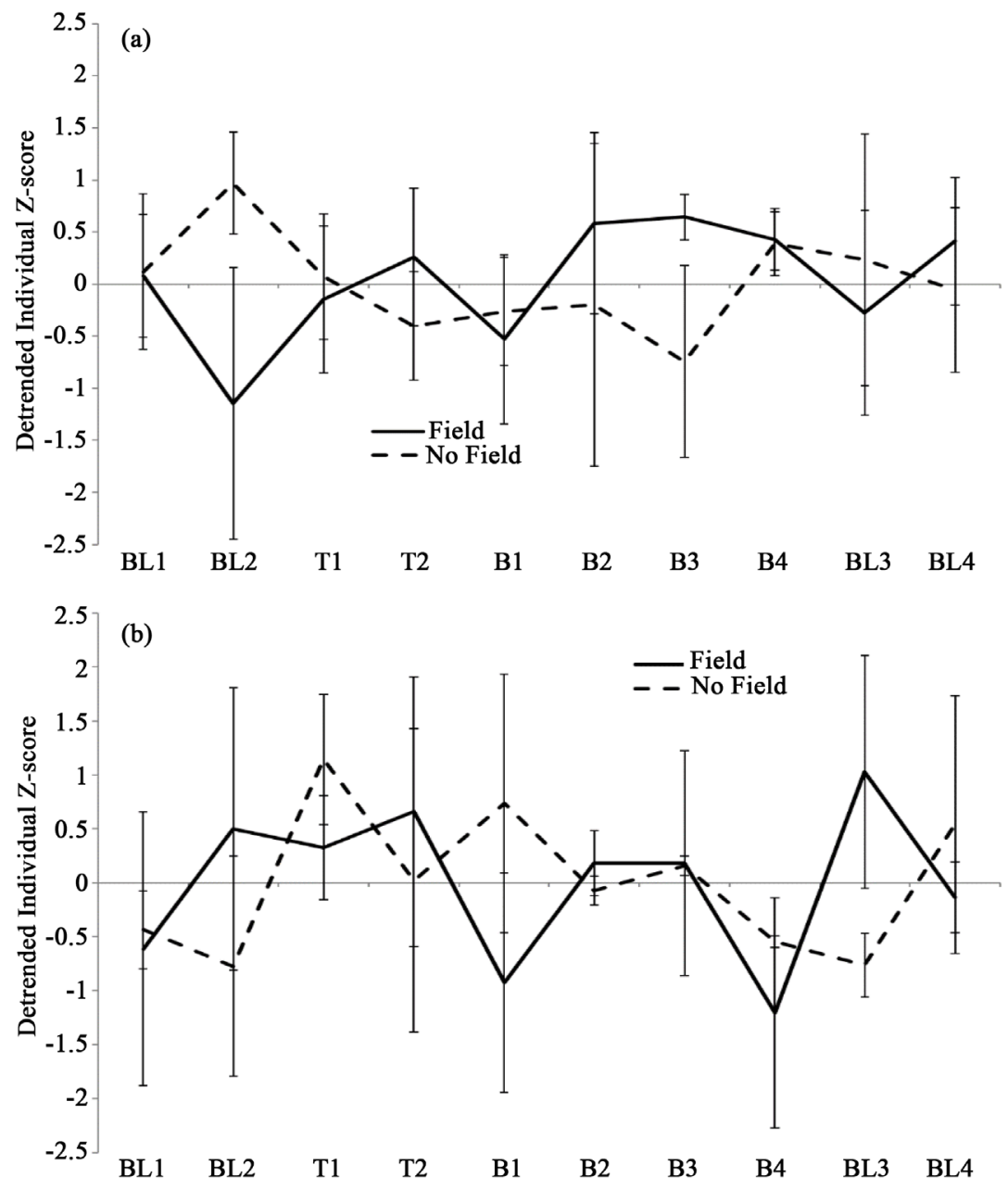

3 (c)

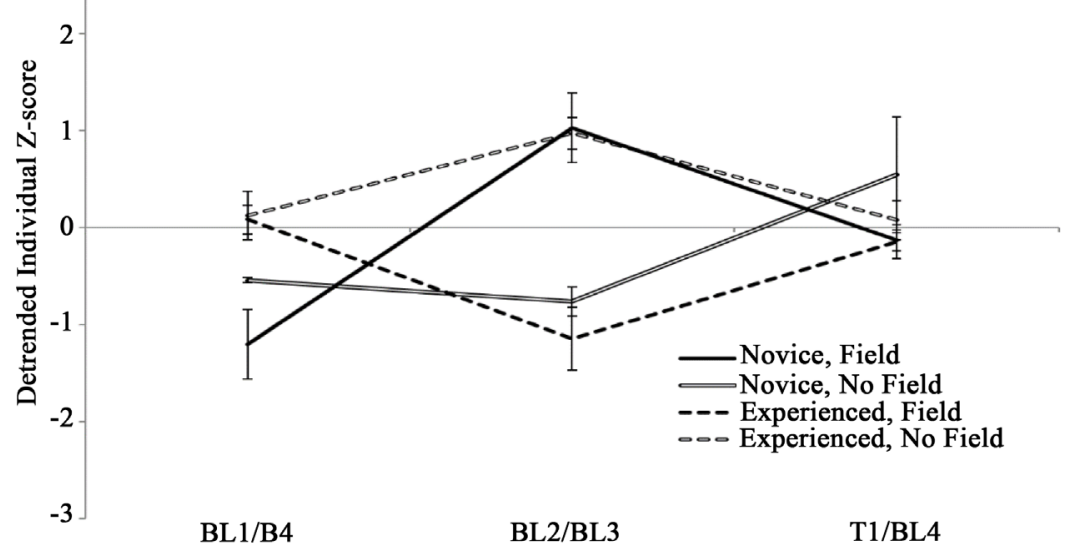

Figure 4. "BL" indicates baseline. "T" indicated Thomas, and "B" indicates BurstX (a) Comparison of detrended individual z-scores between experienced players exposed to fields and experienced players not exposed to fields, with additional breakdown of field exposure time series. Error bars are SD; (b) Comparison of detrended individual z-scores between novice players exposed to fields and novice players not exposed to fields, with additional breakdown of field exposure timeseries. Error bars are SD; (c) Superimposition of detrended individual z-scores between novice players exposed to fields and not exposed to fields at end of experimental time course (B4 - BL4), with detrended individual z-scores between novice players exposed to fields and not exposed to fields at end of experimental time course (BL1 - T1). Error bars are SEM. 
mance level (and by extension) brain activity. Because this is a closed system, however, energy shifts must be accommodated by the law that energy is conserved. Due to the nature of the entanglement of quanta, the maintenance of parity across the time course of the experiment as indicated by Figure 4(c) may provide evidence for temporal non-linearity during the facilitation of such non-locality in space.

\section{Acknowledgements}

Thanks to Dr. Blake Dotta for technical support. The contributions of Viger M. Persinger are appreciated.

\section{References}

[1] Aczel, A.D. (2002) Entanglement: The Greatest Mystery in Physics. Four Walls Eight Windows, New York.

[2] Dotta, B.T. and Persinger, M.A. (2012) "Doubling” of Local Photon Emissions When Two Simultaneous SpatiallySeparated Reactions Share the Same Magnetic Field Configurations. Journal of Biophysical Chemistry, 3, 72-80.

[3] Murugan, N.J., Karbowski, L.M., Dotta, B.T. and Persinger, M.A. (2014) Delayed Shifts in pH Responses to Weak Acids in Spring Water Exposed to Circular Rotating Magnetic Fields: A Narrow Band Intensity-Dependence. International Research Journal of Pure and Applied Chemistry, 5, 131-139. http://dx.doi.org/10.9734/IRJPAC/2015/13156

[4] Scott, M.A., Rouleau, N., Lehman, B.S., Tessaro, L.W., Juden-Kelly, L.M., Saroka, K.S. and Persinger, M.A. (2015) Experimental Production of Excess Correlation across the Atlantic Ocean of Right Hemispheric Theta Pairs Sharing Circumcerebral Rotating Magnetic Fields. Journal of Consciousness Exploration \& Research, 6, 658-684.

[5] Dirac, P. (1930) The Principles of Quantum Mechanics. Oxford University Press, Oxford.

[6] Cavanagh, T. (2012) Super Hexagon.

[7] Dotta, B.T., Buckner, C.A., Lafrenie, R.M. and Persinger, M.A. (2011) Photon Emissions from Human Brain and Cell Culture Exposed to Distally Rotating Magnetic Fields Shared by Separate Light-Stimulated Brains and Cells. Brain Research, 1388, 77-88. http://dx.doi.org/10.1016/j.brainres.2011.03.001

[8] Edelman, G.M. (1989) The Remembered Present. Basic, New York.

[9] Llinas, R. and Ribary, U. (1993) Coherent 40Hz Oscillation Characterizes Dream State in Humans. Proceedings of the National Academy of Sciences, 90, 2078-2081. http://dx.doi.org/10.1073/pnas.90.5.2078

[10] Cook, C.M., Koren, S.A. and Persinger, M.A. (1999) Subjective Time Estimation by Humans Is Increased by Counterclockwise but Not Clockwise Circumcerebral Rotations of Phase-Shifting Magnetic Pulses in the Horizontal Plane. Neuroscience Letters, 268, 61-64. http://dx.doi.org/10.1016/S0304-3940(99)00379-1

[11] Persinger, M.A., Saroka, K.S., Koren, S.A. and St-Pierre, L.S. (2010) The Electromagnetic Induction of Mystical and Altered States within the Laboratory. Journal of Consciousness Exploration \& Research, 1, 808-830.

[12] Booth, J.N. and Persinger, M.A. (2009) Discrete Shifts within the Theta Band between the Frontal and Parietal Regions of the Right Hemisphere and the Experiences of a Sensed Presence. The Journal of Neuropsychiatry \& Clinical Neurosciences, 21, 279-283. http://dx.doi.org/10.1176/jnp.2009.21.3.279

[13] Martin, L.J., Koren, S.A. and Persinger, M.A. (2004) Thermal Analgesic Effects from Weak, Complex Magnetic Fields and Pharmacological Interactions. Pharmacology Biochemistry and Behavior, 78, 217-227. http://dx.doi.org/10.1016/j.pbb.2004.03.016

[14] Juden-Kelly, L.M., Dotta, B.T., Vares, D.A. and Persinger, M.A. (2015) Demonstration of Excess Correlation in NonLocal Random Number Generators Sharing Circular, Changing Angular Velocity Magnetic Fields. Journal of Consciousness Exploration \& Research, 6, 728-737. 


\section{Submit or recommend next manuscript to SCIRP and we will provide best service for you:}

Accepting pre-submission inquiries through Email, Facebook, LinkedIn, Twitter, etc.

A wide selection of journals (inclusive of 9 subjects, more than 200 journals)

Providing 24-hour high-quality service

User-friendly online submission system

Fair and swift peer-review system

Efficient typesetting and proofreading procedure

Display of the result of downloads and visits, as well as the number of cited articles

Maximum dissemination of your research work

Submit your manuscript at: http://papersubmission.scirp.org/ 